This article has been downloaded from IOPscience. Please scroll down to see the full text article. (http://iopscience.iop.org/1355-5111/8/3/025)

More related content is available

Download details:

IP Address: 128.178.55.24

The article was downloaded on 28/11/2007 at 21:29

Please note that terms and conditions apply. 


\title{
Atomic beamsplitter based on multiple adiabatic passage in the optical interference pattern
}

\author{
M Ol'shanii, J Vanicek and M Prentiss \\ Lyman Laboratory, Harvard University, Cambridge, MA 02138, USA \\ Received 4 January 1996
}

\begin{abstract}
We describe a simple and robust method of creating an efficient large-angle adiabatic passage beamsplitter that does not require the light fields to be pulsed. We present simulations that show momentum splittings of $80 \hbar k$, where more than $60 \%$ of the atoms in the initial distribution are in the final momentum peaks at $\pm 40 \hbar k$.
\end{abstract}

\section{Introduction}

There is a great deal of interest in creating an efficient large-angle atomic beamsplitter. It has already been demonstrated that beamsplitters based on adiabatic passage [1] can produce efficient beamsplitting [2-4]. The first demonstrations induced the adiabatic population transfer by allowing atoms to move through a series of partially overlapping laser fields with Gaussian intensity profiles. The intensity of the laser fields was independent of time, but the motion of the atoms through the spatially varying intensity profiles resulted in a series of intensity pulses in the rest frame of the atom. Large splitting angles required that the Gaussian laser fields be passed back and forth through the atomic beam many times, which enhances any imperfections in the optical system and can result in decreased efficiency for splitting angles above a few photon momenta. It has also been shown that adiabatic passage beamsplitting using pulsed light can generate large splitting angles [4]. In this paper we describe a simple and robust method of creating an efficient large-angle adiabatic passage beamsplitter that does not require that the light fields be pulsed. The adiabatic population transfer results from atomic motion through a spatially varying intensity pattern due to optical interference between two laser beams which are nearly copropagating. Thus, a few laser beams can produce hundreds of adiabatic population transfers under nearly ideal optical conditions, resulting in simple, efficient and robust beamsplitting.

In this paper we will present numerical simulations which show momentum splittings of $80 \hbar k$, where more than $60 \%$ of the atoms in the initial distribution are in the final momentum peaks at $\pm 40 \hbar k$. We will discuss the physical mechanisms which result in losses in the beamsplitter and show that analytical predictions based on these mechanisms give excellent agreement with detailed numerical simulations.

\section{Elementary cycle}

In this section we consider a new scheme of adiabatic population transfer which gives a $4 \hbar k$ splitting. We then show (section 3) that multiple repetitions of this scheme can easily be realized by passing an atom through an optical interference pattern. 


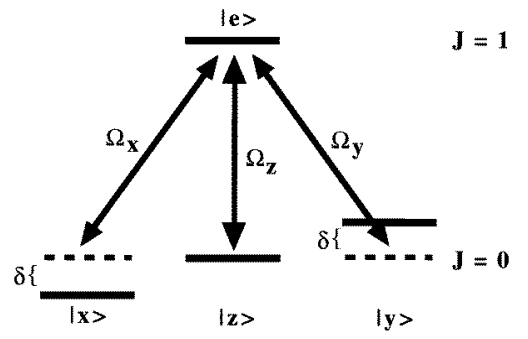

Figure 1. Level scheme for the $J=1 \rightarrow J=0$ transition. $\left|J_{g}=1, \alpha=x, y, z\right\rangle$ and $\left|J_{e}=0, e\right\rangle$ are ground and excited states, respectively. The chemical basis is used instead of the traditional $\left|J_{g}=1, m_{z}=-1,0,+1\right\rangle$ one. The ground states $|x\rangle$ and $|y\rangle$ are assumed to be shifted by $\pm \hbar \delta$ with respect to the $|z\rangle$ state. (For the beamsplitter scheme discussed $\delta=k p_{0} / M$ corresponds to the Doppler shift, $p_{0}$ is the family momentum.) The laser frequency is chosen to be resonant with the $|z\rangle \rightarrow|e\rangle$ transition. $\Omega_{z, x, y}$ are the corresponding Rabi frequencies. In the text we assume the $\Omega_{x}$ and $\Omega_{y}$ Rabi frequencies to be equal: $\Omega_{x}=\Omega_{y}=\Omega_{0}$.

\subsection{Hamiltonian}

Consider the $J_{g}=1 \leftrightarrow J_{e}=0$ transition (figure 1) in the time-dependent laser field

$$
\vec{E}(t)=\overrightarrow{\mathcal{E}}(t) \exp (-\mathrm{i} \omega t)+\mathrm{CC}
$$

where

$$
\overrightarrow{\mathcal{E}}(t)=\sum_{\alpha=x, y, z} \overrightarrow{\mathcal{E}}_{\alpha}(t) \vec{e}_{\alpha} .
$$

When the laser frequency is resonant with the $|z\rangle \rightarrow|e\rangle$ transition $\left(\omega=\omega_{e, z}\right)$, the effective Hamiltonian for such a system has the form

$\hat{H}=-\hbar \delta|x\rangle\langle x|+\hbar \delta| y\rangle\left\langle y\left|+\left\{\sum_{\alpha=x, y, z} \hbar \Omega_{\alpha}(t)|e\rangle\langle\alpha|+\mathrm{HC}\right\}-\frac{\mathrm{i} \hbar}{2} \Gamma\right| e\right\rangle\langle e|$

where

$$
\Omega_{\alpha}=-\langle\|d\|\rangle \mathcal{E}_{\alpha} / \sqrt{3}
$$

are the corresponding Rabi frequencies, $\langle\|d\|\rangle$ is the reduced dipole matrix element, the states $|x\rangle,|y\rangle$ and $|z\rangle$ are given by

$$
\begin{aligned}
& |x\rangle=\frac{-1}{\sqrt{2}}\left(\left|J_{g}=1, m_{z}=+1\right\rangle-\left|J_{g}=1, m_{z}=-1\right\rangle\right) \\
& |y\rangle=\frac{\mathrm{i}}{\sqrt{2}}\left(\left|J_{g}=1, m_{z}=+1\right\rangle+\left|J_{g}=1, m_{z}=-1\right\rangle\right) \\
& |z\rangle=\left|J_{g}=1, m_{z}=0\right\rangle
\end{aligned}
$$

are ground atomic states in the chemical basis, $\Gamma$ is the spontaneous decay rate and $\mp \hbar \delta$ is the energy shift of the $|x\rangle$ and $|y\rangle$ states with respect to $|z\rangle$ (see figure 1).

Assume the laser field to be strong: $|\Omega| \gg \delta, \Gamma$. In this case the four eigenstates of the Hamiltonian (3) are

$$
\begin{aligned}
& |\mathrm{C} \pm(t)\rangle \approx \frac{1}{\sqrt{2}}\left[\sum_{\alpha} n_{\alpha}^{*}(t)|\alpha\rangle \pm|e\rangle\right] \\
& |\mathrm{NC1}, 2(t)\rangle \approx \sum_{\alpha} q_{\alpha}^{1,2}(t)|\alpha\rangle
\end{aligned}
$$


and the corresponding eigenvalues are

$$
\begin{aligned}
& E_{\mathrm{C} \pm}(t) \approx \pm \hbar|\Omega(t)| \\
& E_{\mathrm{NC} 1,2}(t) \approx 0 .
\end{aligned}
$$

Here

$$
\vec{n}=\vec{\Omega} /|\Omega|
$$

is the polarization vector and the vectors $\vec{q}^{1,2}$ must be chosen from the subspace orthogonal $\dagger$ to $\vec{n}^{*}$ :

$$
\vec{q}^{1} \perp \vec{q}^{2} \perp \vec{n}^{*}
$$

Notice the 2D degeneracy for the states $|\mathrm{NC} 1,2\rangle$. These 'non-coupled' states are not shifted by the interaction with the laser light.

\subsection{Adiabatic population transfer in the $J_{g}=1 \leftrightarrow J_{e}=0$ system}

Suppose, that at $t=-\infty$ we prepared our system in some linear combination of the non-coupled states:

$$
|\Psi(-\infty)\rangle=\sum_{\alpha} q_{\alpha}(-\infty)|\alpha\rangle \quad \vec{q}(-\infty) \perp \vec{n}^{*}(-\infty) .
$$

If the field changes slowly enough, the system will always remain in the manifold of the non-coupled states:

$$
|\Psi(t)\rangle=\sum_{\alpha} q_{\alpha}(t)|\alpha\rangle \quad \vec{q}(t)=\hat{U}(t) \vec{q}(-\infty) \quad \vec{q}(t) \perp \vec{n}^{*}(t) .
$$

The unitary matrix $\hat{U}(t)$ is nothing else but the generalized Berry's phase factor described by Wilczek and Zee [5]. Assume the laser field to be real: $\operatorname{Im}(\vec{n})=0$. Then the unitary transformation $\hat{U}(t)$ corresponds to the classical parallel transport of $\vec{q}$ over the surface of the unit sphere, where the trajectory of the motion $\vec{n}(t)$ is defined by the polarization vector (see, for example, [6]).

Now consider the particular cycle shown in figure 2:

$$
\Omega_{z}(t)=\Omega_{0}\left(\frac{t}{\tau}\right) \quad \Omega_{x}(t)=\Omega_{y}=\Omega_{0}
$$

where the trajectory of the polarization vector $\vec{n}$ corresponds to a path from the south pole to the north pole along the $45^{\circ}$ meridian, where $0^{\circ}$ corresponds to the $\pi_{x}$ polarization. The states $|x\rangle$ and $|y\rangle$ are non-coupled states both at the beginning $(t=-\infty)$ and at the end $(t=+\infty)$ of the evolution. The parallel transport law [6] leads to the following transformation rules:

$$
\begin{aligned}
& |\Psi(-\infty)\rangle=|x\rangle \longrightarrow|\Psi(+\infty)\rangle=-|y\rangle \\
& |\Psi(-\infty)\rangle=|y\rangle \longrightarrow|\Psi(+\infty)\rangle=-|x\rangle .
\end{aligned}
$$

Including the Raman detuning $\delta$ in the Hamiltonian (3) does not spoil the transformation rules (15). This can be clearly seen from figure 2 .

$\dagger$ Here and below the scalar product of two complex vectors $\vec{A}$ and $\vec{B}$ is defined as $\langle\vec{A} \mid \vec{B}\rangle=\sum_{\alpha} A_{\alpha}^{*} B_{\alpha}$. 


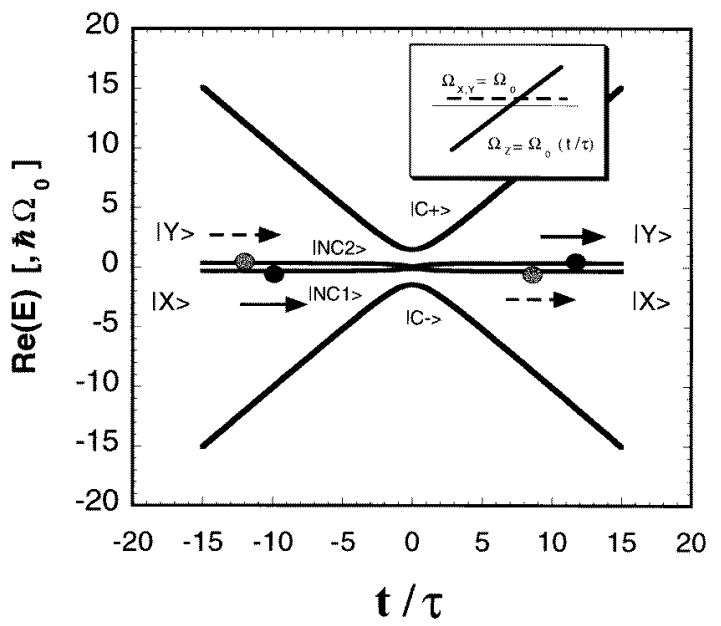

Figure 2. Energy level structure versus time for $\Omega_{x}=\Omega_{y}=\Omega_{0}=$ constant and $\Omega_{z}$ growing linearly with time. Minimum energy splitting between the coupled states $|\mathrm{C}+\rangle$ and $|\mathrm{C}-\rangle$ corresponds approximately to $2 \sqrt{ } 2 \hbar \Omega_{0}$. Splitting between non-coupled states $|\mathrm{NC} 1\rangle$ and $|\mathrm{NC} 2\rangle$ is $2 \hbar \delta$, where $\delta=\Omega_{0} / 3$. The state $|\mathrm{NC} 1\rangle$ connects adiabatically $|x\rangle(t=-\infty)$ and $|y\rangle$ $(t=+\infty)$ bare states, whereas $|\mathrm{NC} 2\rangle$ provides the adiabatic connection between $|y\rangle$ and $|x\rangle$.

\subsection{Elementary cycle in momentum space}

Now consider the spatial motion of the $J_{g}=1 \leftrightarrow J_{e}=0$ system in a laser field

$$
\vec{E}(t)=\overrightarrow{\mathcal{E}}(y, z, t) \exp (-\mathrm{i} \omega t)+\mathrm{CC}
$$

where

$\overrightarrow{\mathcal{E}}(y, z, t)=\mathcal{E}_{x}(t) \exp (+\mathrm{i} k z) \vec{e}_{x}+\mathcal{E}_{y}(t) \exp (-\mathrm{i} k z) \vec{e}_{y}+\mathcal{E}_{z}(t) \exp (\mathrm{i} k y) \vec{e}_{z}$

and $\omega=\omega_{e, z}-\omega_{\mathrm{R}}\left(\omega_{\mathrm{R}}=\hbar^{2} k^{2} / 2 M\right.$ is the recoil energy, $M$ is the atomic mass). In this case the space of the atomic states is split into so-called closed families of states:

$$
\mathcal{F}\left(\vec{p}_{0}\right)=\left\{\left|\vec{p}_{0}, e\right\rangle ;\left|\vec{p}_{0}-\hbar \vec{k}_{z}, x\right\rangle ;\left|\vec{p}_{0}+\hbar \vec{k}_{z}, y\right\rangle ;\left|\vec{p}_{0}-\hbar \vec{k}_{y}, z\right\rangle\right\}
$$

where $\vec{k}_{\alpha}=k \vec{e}_{\alpha}$. Let $\left(p_{0}\right)_{y}=0$ and $\left(p_{0}\right)_{z}=p_{0}$. Then each closed family (18) can be considered as an independent $J_{g}=1 \leftrightarrow J_{e}=0$ system (figure 1), where $\delta=k p_{0} / M$. Corresponding rules for the population transfer due to the cycle (14) are

$$
\begin{aligned}
|\Psi(-\infty)\rangle & =\left|\vec{p}_{0}-\hbar \vec{k}_{z}, x\right\rangle \longrightarrow|\Psi(+\infty)\rangle=-\left|\vec{p}_{0}+\hbar \vec{k}_{z}, y\right\rangle \\
|\Psi(-\infty)\rangle & =\left|\vec{p}_{0}+\hbar \vec{k}_{z}, y\right\rangle \longrightarrow|\Psi(+\infty)\rangle=-\left|\vec{p}_{0}-\hbar \vec{k}_{z}, x\right\rangle .
\end{aligned}
$$

Notice the different directions of the momentum transfer depending on the initial internal state. In section 3 we will use this process (14), (19) as a basic cycle to produce splitting of an atomic beam.

\subsection{Loss channels}

So far we have considered the ideal population transfer process in which $100 \%$ of the atoms are transferred. In this subsection, we will consider the different channels for loss of atoms during the cycle (14). Suppose that we start in the state $|x\rangle$ :

$$
|\Psi(-\infty)\rangle=|\operatorname{NC} 1(-\infty)\rangle=|x\rangle \text {. }
$$


For the ideal cycle $(\delta=0, \Gamma=0, \tau \rightarrow \infty)$ the final state of the atom must be $|y\rangle$. So we define the loss as

$$
\text { loss }=1-|\langle y \mid \Psi(+\infty)\rangle|^{2} .
$$

Below we consider three different channels for the loss. We will calculate the probability for a spontaneous jump (which contains stationary $P_{\mathrm{st}}$ and non-adiabatic $P_{\text {na }}$ parts) as well as the probability of a Landau-Zener transition $P_{\mathrm{L}-\mathrm{Z}}$. The total loss

$$
\text { loss }=P_{\mathrm{st}}+P_{\mathrm{na}}+P_{\mathrm{L}-\mathrm{Z}}
$$

contains all three parts. Note that due to the symmetry between the non-coupled states the formulae for the loss derived below can also be used for the $|y\rangle \rightarrow|x\rangle$ adiabatic passage.

2.4.1. Stationary spontaneous loss. First of all notice that if $\delta \neq 0$ the excited state $|e\rangle$ is always present in the dressed state $|\mathrm{NC} 1(t)\rangle$ at the intermediate stages of the evolution. This gives rise to the spontaneous decays from $|\mathrm{NC} 1(t)\rangle$ even if the motion is very slow. Moreover, the longer the interaction time the more atoms will be lost. The probability of a spontaneous jump will be given by the formula

$$
P_{\mathrm{st}}=\int_{-\infty}^{+\infty} \mathrm{d} t \Gamma_{\mathrm{NC} 1}
$$

where $\Gamma_{\mathrm{NC} 1}=-2 \operatorname{Im}\left(E_{\mathrm{NC} 1}\right)$ is the spontaneous decay rate for the state $|\mathrm{NC} 1\rangle$. To the lowest order in $\delta$ and $\Gamma$

$$
P_{\mathrm{st}}=\xi_{1}\left(\frac{\delta}{\Omega_{0}}\right)^{2} \Gamma \tau
$$

where $\xi_{1}=0.5629$ can be expressed as an integral of a very complicated dimensionless function.

2.4.2. Non-adiabatic spontaneous loss. If the interaction is not slow enough, the atomic state will contain some small fraction of other dressed states. This may also lead to spontaneous decay, even for $\delta=0$. Consider the Hamiltonian (3) in the adiabatic basis:

$$
\hat{H}=\hat{H}_{0}-\hat{A}
$$

where

$$
\left\langle\zeta\left|\hat{H}_{0}\right| \zeta^{\prime}\right\rangle=\delta_{\zeta, \zeta^{\prime}} E_{\zeta}
$$

is the adiabatic Hamiltonian,

$$
\left\langle\zeta|\hat{A}| \zeta^{\prime}\right\rangle=\mathrm{i}\left\langle\zeta \mid(\partial / \partial t) \zeta^{\prime}\right\rangle
$$

is the non-adiabatic coupling between the states and $\zeta=\mathrm{C}+, \mathrm{C}-, \mathrm{NC} 1, \mathrm{NC} 2$. Considering $\hat{A}$ as a small perturbation [7], then we may find a second-order correction $\Gamma_{\mathrm{NC} 1}^{(2)}$ to the spontaneous rate. The probability of the spontaneous jump due to the non-adiabatic corrections is

$$
P_{\mathrm{na}}=\int_{-\infty}^{+\infty} \mathrm{d} t \Gamma_{\mathrm{NC} 1}^{(2)} .
$$

For small $\Gamma$ and $\delta=0$ this probability is given by

$$
P_{\text {na }}=\xi_{2}\left(\frac{1}{\Omega_{0} \tau}\right)^{2} \Gamma \tau
$$

where $\xi_{2}=0.2083$ is a dimensionless constant, which can be expressed in radicals. 
2.4.3. Landau-Zener transitions. Finally, consider the probability of Landau-Zener transitions $P_{\mathrm{L}-\mathrm{Z}}[8]$ from the state $|\mathrm{NC} 1\rangle$ to other dressed states. According to Dykhne's formula [9] we get

$$
P_{\mathrm{L}-\mathrm{Z}}=\sum_{\zeta=\mathrm{C}+\mathrm{C}-} \exp \left[-2 \int_{0}^{\tilde{t}_{\zeta}} \mathrm{d} t \operatorname{Im}\left(E_{\zeta}-E_{\mathrm{NC} 1}\right)\right]
$$

where $\tilde{t_{\zeta}}$ is the crossing point for the $E_{\zeta}(t)$ and $E_{\mathrm{NCl}}(t)$ curves in the complex plane. This leads to the following expression:

$$
P_{\mathrm{L}-\mathrm{Z}}=2 \exp \left[-\pi \Omega_{0} \tau\right]
$$

where $\delta$ and $\Gamma$ are assumed to be small.

\section{Multiple repetition of the elementary cycle in the interference pattern}

In this section we consider multiple repetitions of the basic process (14) and (19) due to the motion through a longitudinal standing wave. This technique allows one to alternate the directions of the laser beams and therefore produce an irreversible splitting of the atomic momentum distribution.

\subsection{Beamsplitter scheme}

Consider the laser field $\left(\omega=\omega_{e, z}-\omega_{\mathrm{R}}\right)$ in the following configuration:

$$
\begin{aligned}
\overrightarrow{\mathcal{E}}(x, y, z)= & \tilde{\mathcal{E}}_{x y} \sin \left(k_{\|} x / 2+\pi / 4\right) \exp (+\mathrm{i} k z) \vec{e}_{x}+\tilde{\mathcal{E}}_{x y} \sin \left(k_{\|} x / 2+\pi / 4\right) \exp (-\mathrm{i} k z) \vec{e}_{y} \\
& +\tilde{\mathcal{E}}_{x y} \sin \left(k_{\|} x / 2-\pi / 4\right) \exp (+\mathrm{i} k z) \vec{e}_{y}+\tilde{\mathcal{E}}_{x y} \sin \left(k_{\|} x / 2-\pi / 4\right) \exp (-\mathrm{i} k z) \vec{e}_{x} \\
& +\tilde{\mathcal{E}}_{z} \cos \left(k_{\|} x\right) \exp (\mathrm{i} k y) \vec{e}_{z}
\end{aligned}
$$

where each longitudinal $(0 X)$ standing wave is the the result of the interference between a corresponding pair of the running waves (see figure $3(a)$ ). For example, the $\pi_{z}$-polarized wave can be built as

$$
\begin{gathered}
\frac{1}{2} \tilde{\mathcal{E}}_{z} \exp [+\mathrm{i} k \cos (\Theta / 2) y+\mathrm{i} k \sin (\Theta / 2) x]+\frac{1}{2} \tilde{\mathcal{E}}_{z} \exp [-\mathrm{i} k \cos (\Theta / 2) y+\mathrm{i} k \sin (\Theta / 2) x] \\
\longrightarrow \tilde{\mathcal{E}}_{z} \cos \left(k_{\|} x\right) \exp \left(\mathrm{i} k_{\perp} y\right) \vec{e}_{z}
\end{gathered}
$$

where

$$
k_{\|} \approx k \Theta / 2 \quad k_{\perp} \approx k
$$

for $\Theta \ll 1$. Let an atomic beam propagate along the $0 X$ axis with a longitudinal velocity $v_{\|}$. In the moving frame atoms will 'see' five transverse running waves with the alternating amplitudes:

$$
\begin{array}{ll}
\Omega_{x}^{\vec{x}}=\tilde{\Omega}_{x y} \sin \left(k_{\|} v_{\|} t / 2+\pi / 4\right) & \Omega_{y}^{\leftarrow}=\tilde{\Omega}_{x y} \sin \left(k_{\|} v_{\|} t / 2+\pi / 4\right) \\
\Omega_{y}^{\overrightarrow{ }}=\tilde{\Omega}_{x y} \sin \left(k_{\|} v_{\|} t / 2-\pi / 4\right) & \Omega_{x}^{\leftarrow}=\tilde{\Omega}_{x y} \sin \left(k_{\|} v_{\|} t / 2-\pi / 4\right) \\
\Omega_{z}=\tilde{\Omega}_{z} \cos \left(k_{\|} v_{\|} t\right) &
\end{array}
$$

where the arrows $\rightarrow, \leftarrow$ represent the $+0 Z$ and $-0 Z$ propagation directions, respectively, and the $\pi_{z}$-polarized wave is propagating along the $0 Y$ axis. 
(a)
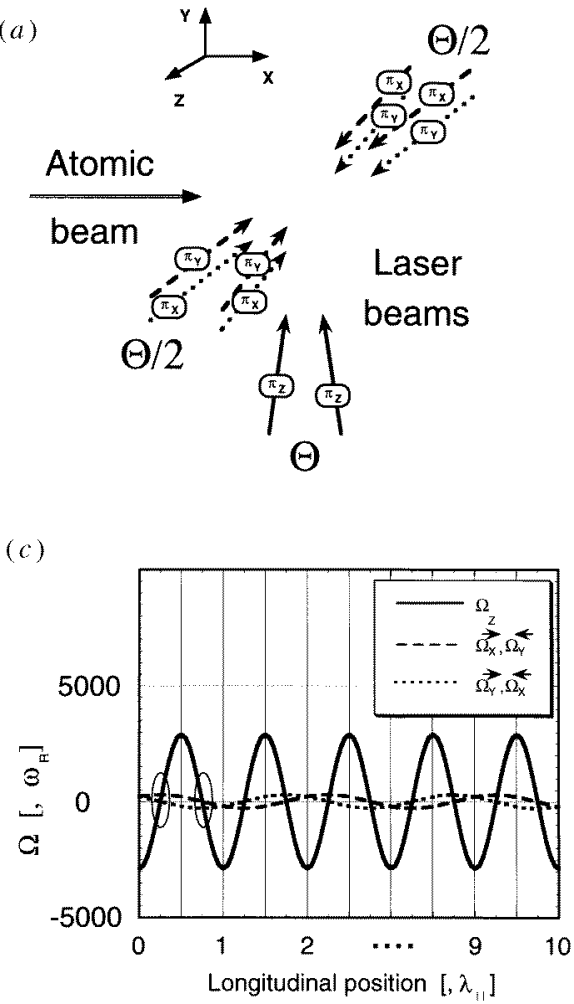

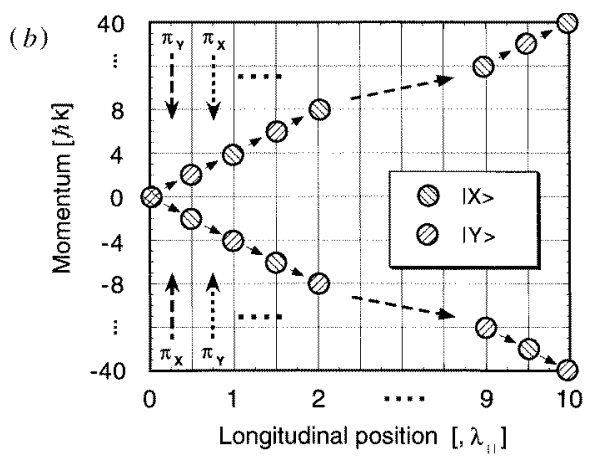

Figure 3. Beamsplitting scheme. (a) Light-field configuration. (b) Field distribution along the atomic beam. Corresponding amplitudes are $\tilde{\Omega}_{x y}=290 \omega_{\mathrm{R}}$ and $\tilde{\Omega}_{z}=2900 \omega_{\mathrm{R}}$. Ellipses show the elementary cycle of figure 2 where $\Omega_{0}=\tilde{\Omega}_{x y}, \tau=\left(\tilde{\Omega}_{x y} / \tilde{\Omega}_{z}\right)\left(k_{\|} v_{\|}\right)^{-1}$, $\delta(n)=2(2 n-1) \omega_{\mathrm{R}}(n=1,2, \ldots, 20$ is the index of the cycle). Each longitudinal standing wave is the result of interference between two running waves with a small angle between them (see $(a)$ ). (c) Schematic diagram for the evolution of the momentum distribution. The initial atomic distribution corresponds to the narrow peak of atoms prepared in the linear superposition of $|x\rangle$ and $|y\rangle$ states. The light-field configuration in the nodes of $\Omega_{z}$ is shown.

Suppose that $\tilde{\Omega}_{z} \gg \tilde{\Omega}_{x y}$. Consider the first node of the $\pi_{z}$-wave (figure $3(b)$ ). If we neglect the $\Omega_{y} \rightarrow \Omega_{x} \leftarrow$ pair of beams as compared to the strong $\Omega_{z}$ wave, the time behaviour of the light field in the vicinity of the node is very similar to the elementary cycle (14), (19). Let the initial state of an atom be

$$
\left|\Psi_{\text {initial }}\right\rangle=\frac{1}{\sqrt{2}}[|\vec{p}=0, x\rangle+|\vec{p}=0, y\rangle]
$$

(see figure 3(c)). Then after passing through the first node, the atomic beam will be split into two parts in accordance with the rules (19). In the next node, the field configuration is similar to one in the first node except for a mirror reflection with respect to the $X 0 Y$ plane. Therefore the interaction with the second node will lead to a further splitting of the beam into two parts separated in momentum space by $8 \hbar k$. Such a sequence may be repeated many times. After passing through $\tilde{n}$ nodes of the $\pi_{z}$-wave, the splitting between two parts of the atomic beam will be $4 \tilde{n} \hbar k$. Different phases of the splitting are shown in figure $3(c)$.

\subsection{Loss of atoms. Monte Carlo simulations}

All three kinds of loss (24), (30) and (33) are accumulated after each elementary cycle. At the end of the evolution, the total loss will be given by the formula

$$
\operatorname{loss}=1-\prod_{n=1}^{\tilde{n}} 1-\left[P_{\mathrm{st}}(n)+P_{\mathrm{na}}+P_{\mathrm{L}-\mathrm{Z}}\right] \approx 1-\exp \left[-P_{\text {sum }}\right]
$$


where

$$
\begin{aligned}
P_{\text {sum }} & =\sum_{n=1}^{\tilde{n}}\left[P_{\mathrm{st}}(n)+P_{\mathrm{na}}+P_{\mathrm{L}-\mathrm{Z}}\right] \\
& \approx \frac{16 \xi_{1}}{3}(\tilde{n})^{3}\left(\frac{\omega_{\mathrm{R}}}{\Omega_{0}}\right)^{2} \Gamma \tau+\xi_{2} \tilde{n}\left(\frac{1}{\Omega_{0} \tau}\right)^{2} \Gamma \tau+2 \tilde{n} \exp \left[-\pi \Omega_{0} \tau\right]
\end{aligned}
$$

and

$$
\begin{aligned}
& \Omega_{0}=\tilde{\Omega}_{x y} \\
& \tau=\left(\tilde{\Omega}_{x y} / \tilde{\Omega}_{z}\right)\left(k_{\|} v_{\|}\right)^{-1} \\
& \delta(n)=2(2 n-1) \omega_{\mathrm{R}} \quad(n=1,2, \ldots, \tilde{n}) .
\end{aligned}
$$

We would like to note that $\tilde{n}$ is the total number of nodes of the $\pi_{z}$-wave.

In figure $4(a)$ and $(b)$ we present the results of quantum Monte Carlo simulations for the metastable $\mathrm{He}$ atoms $\left(\Gamma=38 \omega_{\mathrm{R}}\right)$. In the calculations the momentum space has been truncated to the range $\pm 60 \hbar k$. We have chosen an angle $\Theta=0.053 \mathrm{rad}$, which corresponds to a longitudinal period of the $\pi_{z}$-wave of $\lambda_{\|}=2 \pi / k_{\|}=38 \mu \mathrm{m}$. Corresponding amplitudes are $\tilde{\Omega}_{x y}=290 \omega_{\mathrm{R}}$ and $\tilde{\Omega}_{z}=2900 \omega_{\mathrm{R}}$. The Rabi frequency of the 'strong' wave corresponds to a saturation parameter $s=1.3 \times 10^{6}$. Each plot in figure $4(a)$ and $(b)$ represents the momentum distribution after $\tilde{n}=20$ elementary cycles for longitudinal velocities $5 \mathrm{~m} \mathrm{~s}^{-1}$ and $15 \mathrm{~m} \mathrm{~s}^{-1}\left(3 \omega_{\mathrm{R}} / k_{\|}\right.$and $\left.9 \omega_{\mathrm{R}} / k_{\|}\right)$, respectively. The initial atomic distribution corresponds to the narrow peak of width $\hbar k / 4$. The initial internal state is given by (38). The splitting between the peaks corresponds to the predicted $4 \tilde{n} \hbar k=80 \hbar k$.

In figure 5 the loss of atoms versus longitudinal velocity is shown. We define a transfer as successful if the final momentum $p$ satisfies ||$p|-40 \hbar k| \leqslant \hbar k / 8$. Atoms with any other momentum are considered 'lost'. For the velocity range between $5 \mathrm{~m} \mathrm{~s}^{-1}$ and $15 \mathrm{~m} \mathrm{~s}^{-1}$ more than $60 \%$ of the atoms in the initial distribution are in the final momentum peaks at $\pm 40 \hbar k$.

For comparison we also plot the theoretical prediction (39), (40). The theoretical prediction and the results of the simulations are in good agreement, though no fit parameter has been used. However, the resonance structure of the curve in figure 5 cannot be explained by (39) and (40). Notice that the major resonances correspond to $k_{\|} v_{\|}=1,2,3,4 \omega_{\mathrm{R}}$. They may be associated with Doppler resonances in the residual $\pi_{x}, \pi_{y}$-standing wave.
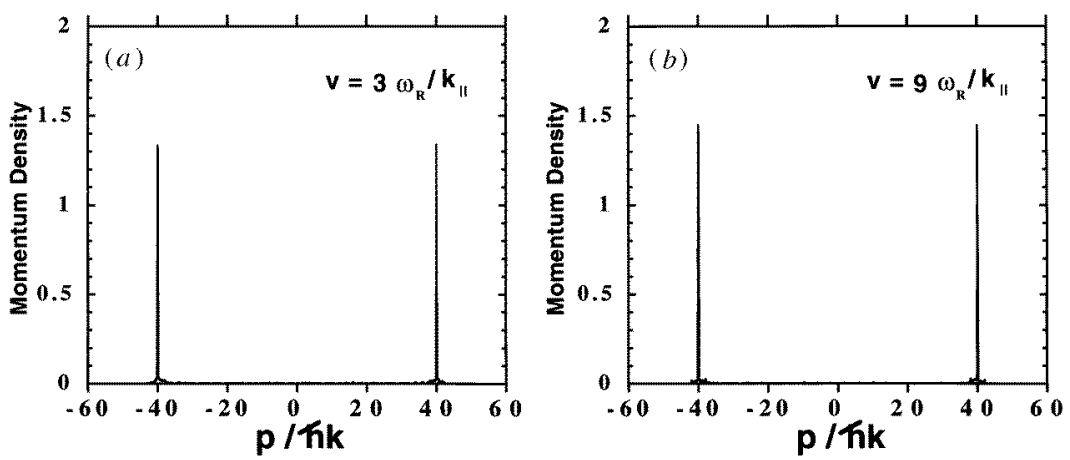

Figure 4. Momentum distribution of metastable $\mathrm{He}$ atoms after the interaction with the beamsplitter (figure 3); $\Theta=0.053 \mathrm{rad}$. The longitudinal velocity is (a) $3 \omega_{\mathrm{R}} / k_{\|}=5 \mathrm{~m} \mathrm{~s}^{-1}$ and (b) $9 \omega_{\mathrm{R}} / k_{\|}=15 \mathrm{~m} \mathrm{~s}^{-1}$, respectively. The initial momentum width of the atomic distribution is $\hbar k / 4$. Each histogram represents an average over 1000 Monte Carlo realizations. 


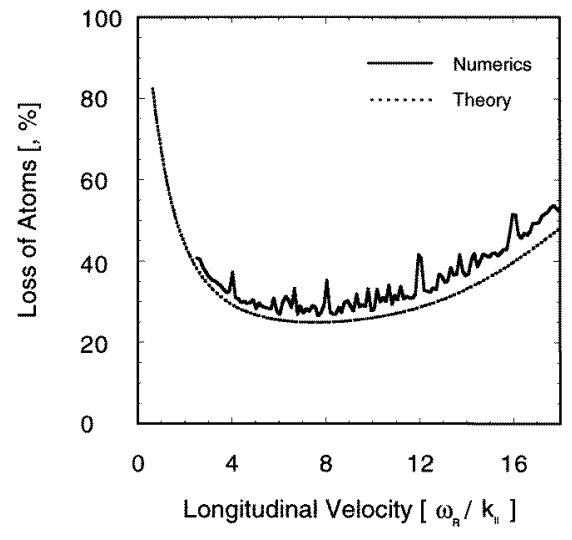

Figure 5. Loss of atoms versus longitudinal velocity. As a desired signal atoms within $\pm \hbar k / 8$ interval around the peaks are considered (see figure 4). Major resonances are located at $k_{\|} v_{\|}=1,2,3,4 \omega_{\mathrm{R}}$. The broken curve corresponds to the analytical prediction (39), (40).

\section{Conclusion}

This paper describes a simple and robust method of creating an efficient large-angle adiabatic passage beamsplitter that does not require pulsed light fields. The beamsplitter generates the population transfer by moving atoms through a spatially varying intensity pattern due to optical interference between two laser beams which are nearly copropagating. We present numerical simulations which show momentum splittings of $80 \hbar k$, where more than $60 \%$ of the atoms in the initial distribution are in the final momentum peaks at $\pm 40 \hbar k$. We show that the loss in the beamsplitter is dominated by three mechanisms: (i) spontaneous emission due to the transverse Doppler shift induced by the beamsplitting; (ii) spontaneous loss due to non-adiabaticity in the population transfer; (iii) Landau-Zener transitions which transfer the atom to undesired dressed states. Analytical predictions based on these mechanisms give excellent correspondence with the results of Monte Carlo simulations.

\section{Acknowledgments}

We acknowledge fruitful discussions with P Marte, J Lawall and C Cohen-Tannoudji. MO was supported by a National Science Foundation grant for the light force dynamics \#PHY93-12572. JV was supported by Harvard University. This work was partially supported by the NSF through a grant for the Institute for Theoretical Atomic and Molecular Physics at Harvard University and Smithsonian Astrophysical Observatory.

\section{References}

[1] Marte P, Zoller P and Hall J L 1991 Phys. Rev. A 444118

[2] Lawall J and Prentiss M 1994 Phys. Rev. Lett. 72993

[3] Goldiner L S, Gerz G, Spreew R J C, Rolston S L, Westbrook C I, Phillips W D, Marte P and Zoller P 1994 Phys. Rev. Lett. 72997

[4] Weitz M, Young B C and Chu S 1994 Phys. Rev. Lett. 722563

[5] Wilczek F and Zee A 1984 Phys. Rev. Lett. 522111

[6] Berry M V 1989 Geometric Phases in Physics ed A Shapere and F Wilczek (Singapore: World Scientific) p 7

[7] Moody J, Shapere A and Wilczek F 1989 Geometric Phases in Physics (Singapore: World Scientific) p 160

[8] Zener C 1932 Proc. R. Soc. A 137696

[9] Dykhne A M 1962 JETP 14941 